\title{
ESS super beam for neutrino CP violation discovery
}

\author{
Tord Ekelöf ${ }^{1}$ \\ Department of Physics and Astronomy, Uppsala University \\ Box 516, SE-75120 Uppsala, Sweden \\ E-mail: Tord.Ekelof@physics.uu.se
}

\begin{abstract}
$\underline{\text { Abstract }}$
The linear proton accelerator of the European Spallation Source (ESS) has the potential to become the proton driver of - in addition to the world's most intense pulsed spallation neutron source - the world's most intense neutrino beam. The physics performance of this neutrino beam in conjunction with a megaton water Cherenkov neutrino detector installed $1000 \mathrm{~m}$ down in a mine at a distance of ca $500 \mathrm{~km}$ from ESS, corresponding to the position of the second neutrino oscillation maximum, is described. The superior potential of such a neutrino experiment for lepton CP violation discovery, with the prospect of explaining the matter-antimatter asymmetry in Universe, is discussed. The equipment required, in particular radiofrequency sources of increased power, an accumulator ring and a neutrino target, to operate the ESS linac for neutrino beam production in parallel with neutron production, is also discussed.
\end{abstract}

XV Workshop on Neutrino Telescopes

March 11-15, 2013

Venice, Italy

\footnotetext{
${ }^{1}$ Speaker
} 


\section{Introduction}

We assume that when matter and antimatter were created from energy in the Big Bang they appeared in equal amounts, like we see that matter is always produced in particleantiparticle pairs at our high energy physics accelerators. However, in the Universe we observe today the amount of matter is enormously much more abundant than antimatter. The amount of $\mathrm{CP}$ violation observed in the quark sector is too small to explain this abundance of matter over antimatter through hadrogenesis $[1,2]$. If $\mathrm{CP}$ violation were to be observed in the neutrino sector, this would open the possibility to explain the matter abundance in Universe through the mechanism of leptogenesis [3]. This makes the discovery of neutrino CP violation a high priority goal in today's particle physics research. In the neutrino sector CP violation is best searched for in $v_{\mu} \rightarrow v_{\mathrm{e}}$ oscillations, which can be studied by creating a $v_{\mu}$ beam from a high intensity proton beam hitting a target, thus producing charged $\pi$ 's that decay to $\mu+v_{\mu}$, and using a large mass neutrino detector at a long distance to measure the appearance of $v_{\mathrm{e}}$.

As neutrino cross-sections are very small, very intense neutrino beams, and thus very intense proton beams, are required for such measurements. The proton linear accelerator (linac) of the European Spallation Source (ESS) in Lund in southern Sweden is currently entering its construction phase and will be the world's most intense proton accelerator, accelerating more than $10^{23}$ protons er year to 2 or $2.5 \mathrm{GeV}$ energy. Initial operation of the ESS linac will start in 2019 and full operation in 2022. We propose [4] to make use of this accelerator to also produce the world's most intense neutrino beam with the purpose of searching for neutrino CP violation. The method we propose for this is such that it will not reduce or affect in any other way the concurrent use of the ESS accelerator to produce spallation neutrons.

\section{The use of the ESS linac}

The ESS proton accelerator is a superconducting linac designed to accelerate $2.86 \mathrm{~ms}$ long $50 \mathrm{~mA}$ proton pulses to $2.5 \mathrm{GeV}$. Figure 1 shows a schematic picture of the different components of the $450 \mathrm{~m}$ long linac; the proton source, the low energy beam transport (LEBT), the radio frequency quadrupole (RFQ), the medium energy beam transport (MEBT), the drift tube linac (DTL), the spoke accelerating cavity section, the low and high velocity ( $\beta$ ) elliptical accelerating cavity sections and a $100 \mathrm{~m}$ long high energy beam transport (HEBT) section to leave space in the accelerator tunnel for future upgrades.

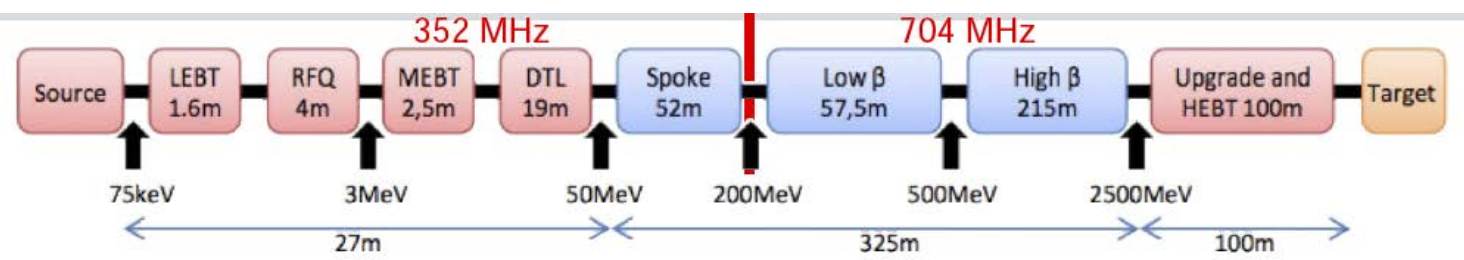

Figure 1. Schematic lay-out of the ESS linac.

It is foreseen to operate this linac at $14 \mathrm{~Hz}$ pulse frequency for protons to be sent to the spallation target, which implies a beam power of $5 \mathrm{MW}$. We are proposing to double the pulse frequency to $28 \mathrm{~Hz}$, which will raise the beam power to $10 \mathrm{MW}$, guiding every second pulse to 
a neutrino target, thus providing concurrent production of spallation neutrons and a neutrino Super Beam. For this the average capacity of the linac radiofrequency (RF) power sources, as well as that of the linac cooling system, will need to be doubled. As in this way the instantaneous RF power level is not increased, the upgrade of the RF power sources will essentially consist in doubling the capacity of their anode current supplies such that the average current to be drawn from the anodes can be doubled. For this it will be essential that the possibility to add a second anode power supply to, or to double the capacity of the original supply of, each RF power supply be foreseen when ordering the power supplies in industry, which is planned for as from 2016, and that the cooling system of the accelerating cavities and their power couplers be such that it can accept a doubling of the coolant flow.

\section{The neutrino target station}

The cylindrical target to be hit by the proton beam must have a sufficiently small radius, ca $1 \mathrm{~cm}$, such that the produced charged pions can leave the target sideways, get focused in the forward direction by a magnetic horn and then decay to produce $\mu+v_{\mathrm{e}}$ pairs in a decay tunnel, which will be about $25 \mathrm{~m}$ long, ending with a beam stopper that will absorb the $\mu$ 's before they start decaying to $\mathrm{e}+v_{\mu}+v_{\mathrm{e}}$ in order to avoid a significant contamination of $v_{\mathrm{e}}$ 's in the initial $v_{\mu}$ beam. There are several challenging problems with the target station which are due to the exceptionally high beam power; the beam heat dissipation in the target, the electrical heat dissipation in the current leads of the focusing magnetic horn and the induced radiation in the target area and the decay tunnel.

In order to reduce the target heating it is proposed to divide the target-horn assembly up on four, which will reduce the average beam power hitting each of the four target-horn assemblies to $1.25 \mathrm{MW}$ beam. The current design of the targets is a tube containing ca $0.1 \mathrm{~mm}$ diameter beads of titanium that are cooled with a very high velocity flow of cold Helium gas. A prototype of this design will be tested in the HiRadMat intensity beam testing area at CERN [5, 6]. In order to reduce the electrical heat dissipation in the current-leads of the horns, the length of the current pulses sent from the pulse generator to the horns must be limited to a few microseconds. This implies that also the beam pulses must be that short. In order to shorten the $2.86 \mathrm{~ms}$ long pulses in the linac to $1.5 \mu \mathrm{s}$, a beam accumulator ring will be needed. This ring is described in the next section. To contain the induced radiation in the target area and the decay tunnel they must be located ca $25 \mathrm{~m}$ underground and be surrounded by thick concrete walls as well as air and water tight envelopes.

\section{The accumulator and the $\mathrm{H}^{-}$source}

As noted in the previous section the $2.86 \mathrm{~ms}$ long linac beam pulse needs to be compressed to $1.5 \mu \mathrm{s}$. This is achieved by multi-turn injection of the linac beam pulse into a $450 \mathrm{~m}$ circumference storage ring followed by a single turn ejection. The primary challenge of the design of such an accumulator ring is the problems caused by the large space charge effects of the ca $10^{15}$ protons stored in the ring for each pulse. Also in this case, the proposal is to reduce the problem by dividing up the storage ring on four rings, each then having to contain 
only a quarter of the full charge. To realize this, the beam pulse from the linac will be split up on four beams that will be led to the four accumulation rings and then further to the four targets.

Even so, with proton injection into the rings the beam pulse charge to be stored is too high to maintain stable beam conditions in the compressor rings and it will therefore be necessary to use $\mathrm{H}^{-}$ions to inject in the accumulator rings, stripping these ions of their two electrons using strong magnetic fields and a laser at the point where the ions meet the stored circulating proton beam in the compressor ring. This in turn implies that the pulses in the linac that are intended for neutrino production must be $\mathrm{H}^{-}$pulses. To realize this there must be a $\mathrm{H}^{-}$ ion source added to the proton source of the linac. $\mathrm{H}^{-}$ions has already been used in other accelerators, like e.g. the SNS in the U.S. The current idea for the ESS linac is that the $\mathrm{H}^{-}$and proton beam lines from the respective sources will merge in the accelerator just upstream of the RFQ. The injection of the negative $\mathrm{H}^{-}$micro-pulses will have to be phase shifted with respect to the injection of the positive proton micro-pulses. The phase shifting scheme will also have to cope with the fact that there are two different radiofrequencies used in the linac, $352 \mathrm{MHz}$ up to the spoke cavity section and $704 \mathrm{MHz}$ beyond that section. It may be noted that some of the spallation neutron users are in need of neutron pulses significantly shorter than $2.86 \mathrm{~ms}$ and that the compressor ring will be of use also for the spallation neutron research community.

\section{The optimal neutrino beam base line}

The neutrinos produced by the ESS proton beam will have a mean energy of about 350 $\mathrm{MeV}$. It is of primary importance to place the large neutrino detector at the position from the neutrino source in Lund which is optimal for CP violation. For $350 \mathrm{MeV}$ the first $v_{\mu} \rightarrow v_{e}$ oscillation maximum is at ca $150 \mathrm{~km}$ distance and the second at ca $450 \mathrm{~km}$ distance. Until 2012 it was assumed that the, at that time, unmeasured neutrino mixing angle $\theta_{13}$ was small. Under that assumption the optimal sensitivity to CP violation is at the first oscillation maximum. All other currently discussed neutrino long base-line projects, like the US LBNE project [7, 8, 9], the European LBNO project [10] and the Japanese HyperK project [11], had their neutrino energy and baseline chosen well before 2012 and such that the respective detectors are positioned at the first neutrino oscillation maximum.

However, in 2012 several high precision neutrino experiments managed to measure the value of $\sin ^{2}\left(2 \theta_{13)}\right.$ to be about 0.1 which is much higher than earlier assumed. At this high value the sensitivity to CP violation is significantly higher at the second $v_{\mu} \rightarrow v_{\mathrm{e}}$ oscillation maximum than at the first [12]. In view of this the distance chosen for the ESS-based neutrino experiment is about $450 \mathrm{~km}$. Now, the neutrino energy distribution around $350 \mathrm{MeV}$ is broad, its width is about as large as it mean value as can be seen in figure 2, which shows the energy distribution for detected oscillated electron neutrinos and antineutrinos, respectively, plus backgrounds. In order to make a more accurate determination of the optimal baseline, a full simulation of the experiment based on the GLoBES $[13,14,15]$ code has been made of the fraction of the full CP phase angular range within which a $3 \sigma$ and $5 \sigma$ discovery of CP violation could be made as function of the baseline. The results are shown in figure 3 for three different proton energies. These curves confirm that it is at the position of the second oscillation maximum ( 450 km), and not at that of the first ( $150 \mathrm{~km})$, that the sensitivity to CP violation is at its maximum. 

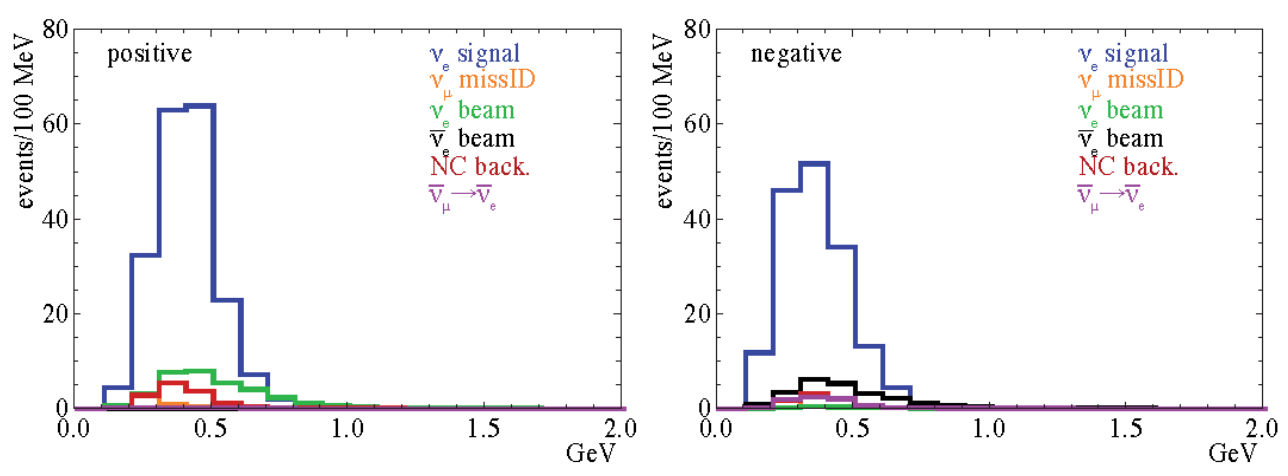

Figure 2. The energy distribution for detected oscillated electron neutrinos and antineutrinos, respectively, and for the different backgrounds.

It may be noted that for the three other planned long base-line projects mentioned above it is not so easy to adjust to the new situation with a high measured value of $\theta_{13}$, since this would imply either to move the detector to a site which is ca 3 times further away from the neutrino source or to reduce the neutrino energy by a factor 3 . If a new underground site were to be available at the required bigger distance (which for none of the three projects mentioned is obviously the case) the proton power would have to be significantly increased to compensate for the smaller solid angle detector acceptance. To reduce the neutrino-beam energy by a factor 3 would imply that the beam energy of the proton accelerator would have to be reduced by a factor significantly larger than 3 , which is very difficult to achieve without a substantial loss in proton intensity for the three projects mentioned, as all of them use circular proton accelerators.

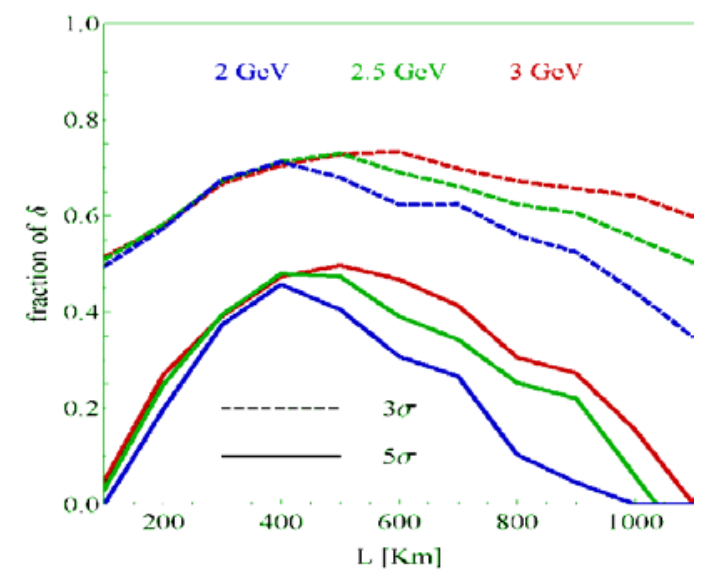

Figure 3. The fraction of the full CP phase angular range within which a $3 \sigma$ and $5 \sigma$ discovery of CP violation can be made for 3 different proton beam energies as function of the baseline.

\section{The neutrino detector underground site}

In view of the low detection cross-sections for neutrinos of about $350 \mathrm{MeV}$ and the inevitable increase of the transverse neutrino-beam size with distance, the target mass of the detector to be used for the ESS neutrino project must be very large, i.e. as it turns out, of the 
order of a megaton. For such a large target mass the water Cherenkov detector is the only option. The detection capabilities of this type of detector is sufficient for the ESS neutrino beam as the background from inelastic neutral-current events, containing several charged particle tracks in the final state, is relatively seen very low for a $350 \mathrm{MeV}$ neutrino beam.

A water Cherenkov detector with a fiducial detector mass of half a megaton, which is ca 20 times the fiducial mass of the current SuperKamiokande water Cherenkov detector, also has unique capabilities for measurements of supernova neutrinos, atmospheric neutrinos, solar neutrinos and proton decay. Unlike for the measurements of the 14 accelerator neutrino beam pulses/second, each of length $1.5 \mu$ s, these other measurements will need the detector to be continuously sensitive. For this reason the detector must be located at least $1000 \mathrm{~m}$ underground or the cosmic ray background would completely obscure these other measurements.

In figure 4 is shown a map of Scandinavia with the locations of several deep mines marked, with, for each mine, the distance to ESS in Lund and the depth indicated. From the curves in figure 3 it may be seen that it is the Garpenberg and Zinkgruvan mines at 540 and 365 $\mathrm{km}$ distance from Lund that are of prime interest as neutrino detector sites. Of these, the Garpenberg mine presents the advantage of that one of its currently used shafts with its ore hoist will no longer be used for mining operations after 2014 and could thus be used to bring up the scrap rock from the excavation of the large water-Cherenkov detector cavern. The capacity of the hoist is $110^{\prime} 000 \mathrm{~m}^{3}$ rock debris per year, which implies that 600 ' $000 \mathrm{~m}^{3}$ cavern can be excavated in about 5 years.

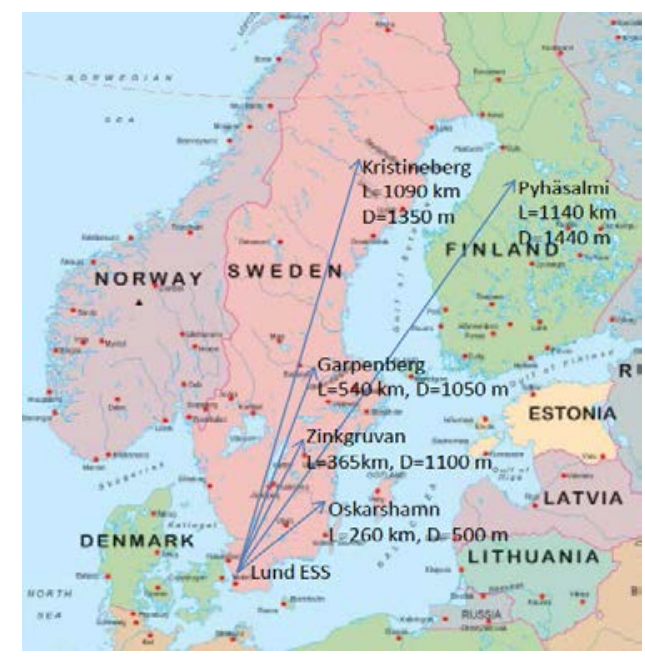

Figure 4. A map of Scandinavia with the location of several deep mines marked with, for each mine, the distance L to ESS in Lund and the depth D indicated.

The dominating limestone and dolomite rock in the ca $2 \mathrm{~km}$ broad and $10 \mathrm{~km}$ long syncline ( area) of the Garpenberg mine is not suitable for the excavation of the very large detector cavern. However, the syncline is surrounded by granite which is of sufficient strength to allow the safe construction of large caverns. Bore holes have been made out to the granite zone and recent rock strength measurements of 18 granite bore core samples have yielded a mean value of the uniaxial compressive strength of $206 \mathrm{MPa}$. The rock stress at $880 \mathrm{~m}$ depth has previously been measured. The main component of the stress is $40 \mathrm{MPa}$ horizontal and 
directed perpendicularly to the syncline. These values indicate that caverns of the size required for the large neutrino detector can be constructed in the granite zone.

\section{Performance for CP violation discovery}

By placing the neutrino detector of the ESS neutrino project at the second oscillation maximum the project has been optimized for CP violation discovery. The other remaining neutrino parameter to be determined is that of the mass hierarchy, i.e. to find out whether the mass-eigenstate neutrino $v_{3}$ is heavier (normal hierarch, $\mathrm{NH}$ ) or lighter (inverted hierarchy, $\mathrm{IH}$ ) than the other two mass-eigenstates $v_{1}$ and $v_{2}$. The sensitivity of a measurement of the mass hierarchy is highest at the first oscillation maximum and with long neutrino beam baselines, for which the mass effect is large. The ESS neutrino project can be used for mass hierarchy measurements using atmospheric neutrinos. However, there are several other existing and planned experiments that are more optimized for mass hierarchy determination than for CPviolation discovery in addition to LBNE, LBNO and HyperK, and it is quite probable the mass hierarchy will be determined within a decade from now.

Using the GLoBES program, the performance for CP violation discovery of the ESS neutrino project and other proposed long baseline neutrino projects has been evaluated. The results are shown in figures 5 and 6 . In figure 5 is shown the significance in terms of number of standard deviations with which CP violation can be discovered as function of the fraction of the full range of the $\mathrm{CP}$ angle $\delta_{\mathrm{CP}}$ for different proposed experiments. For the ESS neutrino project (ESSvSB in the figures) results for two different baselines of $360 \mathrm{~km}$ and $540 \mathrm{~km}$ are shown together with the results for LBNE, LBNO and HyperK. "2020" considers 3+3 years of NOvA, and 5 years only for neutrinos in T2K (at its nominal luminosity, $0.75 \mathrm{MW}$ ); "2025" considers $5+5$ years of NOvA, and $5+5$ years for T2K. Figure 6 shows the fraction of the full $\delta_{\mathrm{CP}}$ range for which an error in the angle $\delta_{\mathrm{CP}}$ (at $1 \sigma, 1$ degree of freedom ) or better could be achieved. The results shown are for the same experimental projects as in figure 5 .

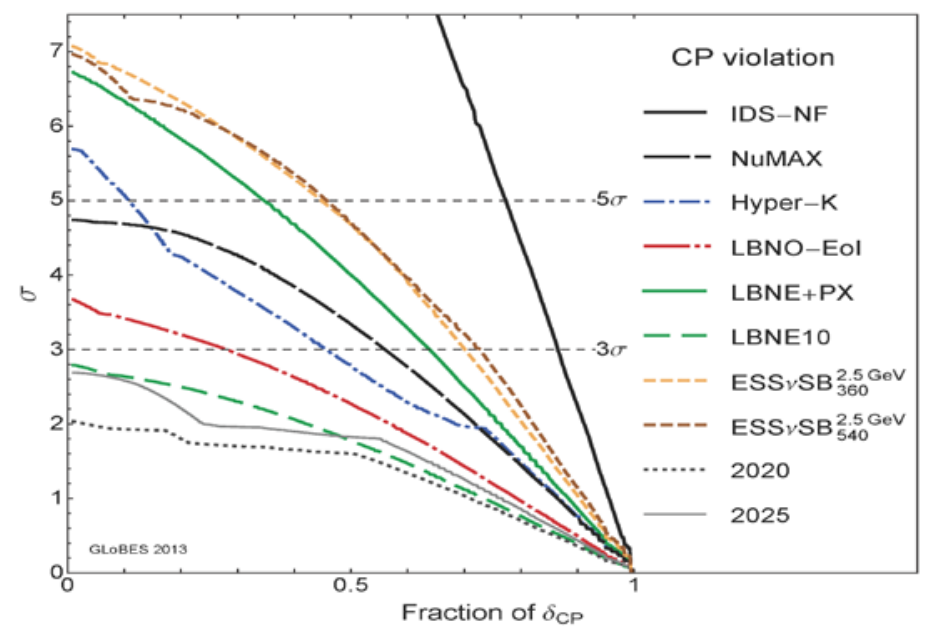

Figure 5. The significance in terms of number of standard deviations with which CP violation can be discovered as function of the fraction of the full range of the $\mathrm{CP}$ angle $\delta_{\mathrm{CP}}$ for different proposed experiments. 


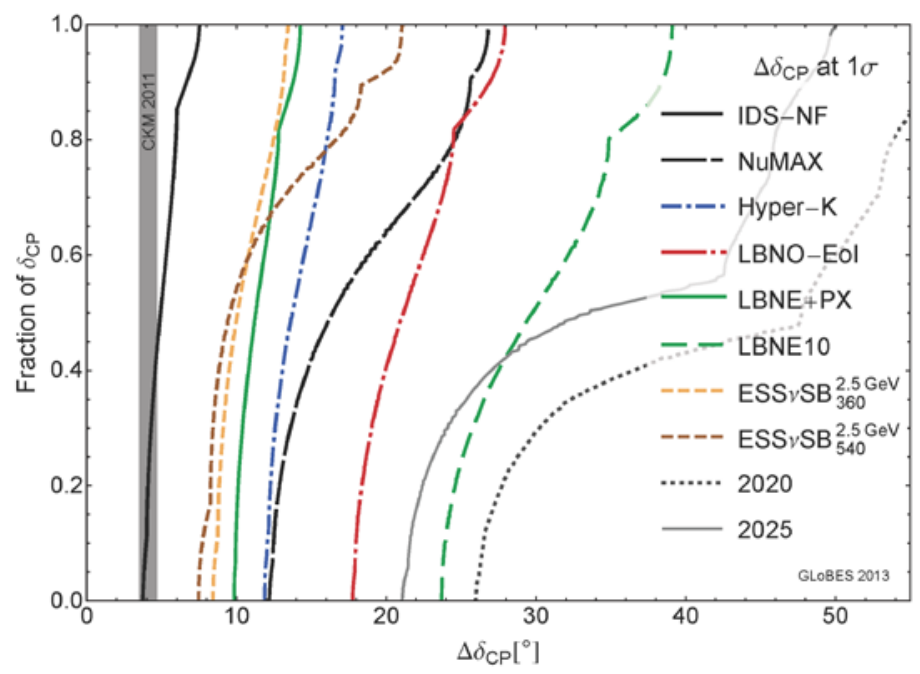

Figure 6. The fraction of the full $\delta_{\mathrm{CP}}$ range for which an error $\Delta \delta_{\mathrm{CP}}$ in the CP angle (at $1 \sigma, 1$ degree of fredome ) or better could be achieved.

Both the curves in figure 5 and those in figure 6 show that the ESS neutrino project comes out as having the best performances among the currently proposed projects, only surpassed by the Neutrino Factory, which builds on the use of, as of today, unproven accelerator techniques, in particular muon cooling and circulation in a storage ring. The reason for the high performance of the ESS neutrino project is to be sought in the combination of:

- the detector position at the second neutrino oscillation maximum, where the sensitivity to the $\mathrm{CP}$ angle is significantly enhanced as compare to the first maximum,

- the low neutrino energy, implying a short oscillation wave length, leading to a short base line even for the second maximum and thereby negligible influence of the mass effect, and

- a very high flux of neutrinos, which more than compensates for the loss of event statistics due to the low neutrino cross-sections at low energy and to the loss in detector acceptance due to the widening of the neutrino beam over the 3 times longer baseline at the second oscillation maximum as compared to the first maximum.

\section{Concluding remarks}

Using the ESS linac as proton driver for a neutrino Super Beam will provide world competitive capability to search for CP violation in the neutrino sector. To further demonstrate the feasibility of this project, tests will be needed of the proposed method to double the ESS pulse frequency, and thereby the power, with the purpose of enabling the production of a uniquely intense neutrino beam concurrently with the production of spallation neutrons. Furthermore the method to accelerate and inject $\mathrm{H}^{-}$ions in a $450 \mathrm{~m}$ circumference storage ring to compress the $2.86 \mathrm{~ms}$ long linac pulses to $1.5 \mu \mathrm{s}$, employing a laser pulse to strip the $\mathrm{H}$ - ions at injection into the ring, needs testing. Also the use of a very small radius helium-gas-cooled cylindrical canister filled with titanium beads as target and the generation and sustainability of very intense current pulses in the neutrino horns to focus the pion flux will 
have to be further investigated. In addition, the conditions and methods for excavating very large detector cavern in the granite at an existing mine located about $500 \mathrm{~km}$ from ESS in Lund, like the Garpenberg mine, will need further exploration. Preliminary studies of all these conditions have until now encountered no fundamental problem and the prospects for the realization of a world leading ESS neutrino project ESSnuSB appear as very promising.

\section{References}

[1] M. B. Gavela, P. Hernandez, J. Orlo and O. Pene, Mod. Phys. Lett. A 9 (1994) 795[hep$\mathrm{ph} / 9312215]$

[2] M. B. Gavela, P. Hernandez, J. Orlo_, O. Pene and C. Quimbay, Nucl. Phys. B 430 (1994)382 [hep-ph/9406289]

[3] F.-X. Josse-Michaux; Recent developments in thermal leptogenesis: the role of favours in various seesaw realizations; arXiv:0809.4960v1

[4] E. Baussan et al,. A Very Intense Neutrino Super Beam Experiment for Leptonic CP Violation Discovery based on the European Spallation Source Linac: A Snowmass 2013 White Paper; arXiv:1309.7022v3

[5] H. Gaillard, C. Theis, H. Vincke, I. Efthymiopoulos, S. Evrard, D. Grenier, A. Pardons andP. Trilhe et al., Conf. Proc. C 110904 (2011) 1665.

[6] High-Radiation to Materials (HiRadMat) Facility of CERN/SPS,https://espace.cern.ch/hiradmatsps/WikiPages/Home.aspx

[7] LBNE Conceptual Design Report from Oct 2012, volume 1, https://sharepoint.fnal.gov/project/lbne/LBNE\%20at\%20Work/SitePages/Reports\%20and\%20Docum ents.aspx.

[8] T. Akiri et al. [LBNE Collaboration], arXiv:1110.6249 [hep-ex].

[9] C. Adams et al. [LBNE Collaboration], arXiv:1307.7335 [hep-ex].

[10] A. Stahl, C. Wiebusch, A.M. Guler, M. Kamiscioglu, R. Sever, et al. \Expression of Interestfor a very long baseline neutrino oscillation experiment (LBNO)", (2012).

[11] K.Abe et al. Letter of Intent: The Hyper-Kamiokande Experiment --- Detector Design and Physics Potential ---, arXiv:1109.3262 [hep-ex]

[12] S.Parke Neutrinos: Theory and Phenomenology arXiv:1310.5992v1 [hep-ph] 22 Oct 2013 , see in particular page 12

[13] P. Coloma, P. Huber, J. Kopp and W. Winter, Phys. Rev. D 87 (2013) 3, 033004[arXiv:1209.5973 [hep-ph]]

[14] P. Huber, M. Lindner and W. Winter, Comput. Phys. Commun. 167 (2005) 195[hep$\mathrm{ph} / 0407333]$

[15] P. Huber, J. Kopp, M. Lindner, M. Rolinec and W. Winter, Comput. Phys. Commun. 177(2007) 432 [hep-ph/0701187] 